06

\title{
Микроволновый нагрев металлических порошковых кластеров
}

\author{
(C) К.И. Рыбаков, ${ }^{1,2}$ В.Е. Семенов, ${ }^{1}$ И.И. Волковская ${ }^{1,2}$ \\ ${ }^{1}$ Институт прикладной фризики РАН, \\ 603950 Нижний Новгород, Россия \\ ${ }^{2}$ Нижегородский государственный университет им. Н.И. Лобачевского, \\ 603000 Нижний Новгород, Россия \\ e-mail: rybakov@appl.sci-nnov.ru
}

\section{Поступило в Редакцию 22 апреля 2017 г.}

\begin{abstract}
Приведены результаты моделирования высокоскоростного микроволнового нагрева сферических кластеров из металлических частиц до температуры плавления. При моделировании рассматривается кластер в поле плоской электромагнитной волны. Размер кластера сопоставим с длиной волны, а учет возмущений поля внутри кластера выполняется в приближении эффективной среды. Показано, что при напряженностях поля в падающей волне порядка $2 \mathrm{kV} / \mathrm{cm}$ на частоте $24 \mathrm{GHz}$ и $5 \mathrm{kV} / \mathrm{cm}$ на частоте $2.45 \mathrm{GHz}$ время нагрева в вакууме до температуры плавления не превышает $1 \mathrm{~s}$. Полученные результаты позволяют сделать вывод о том, что применение высокоскоростного микроволнового нагрева позволяет реализовать сфероидизацию металлических частиц в целях получения высококачественных порошков для аддитивных технологий.
\end{abstract}

DOI: $10.21883 / J T F .2018 .01 .45480 .2307$

В последние годы интенсивное развитие получили исследования в области аддитивных технологий изготовления изделий. В отличие от традиционной технологии изготовления изделий путем механической обработки с удалением из массива заготовки лишнего материала, аддитивные методы основаны на наращивании изделия путем послойной консолидации (сплавления, спекания и т.д.) исходного конструкционного материала (порошка) при локализованном термическом воздействии согласно заранее созданной трехмерной модели изделия. Применение аддитивных методов особенно перспективно в тех случаях, когда изготовление изделий носит штучный или малосерийный характер, как например, в задачах восстановительной медицины или при изготовлении изделий аэрокосмической техники, поскольку делает возможным синтез изделий сложной формы и/или изделий с изменяющимся по объему композиционным составом.

Отдельной проблемой при реализации аддитивных методов изготовления изделий является получение металлических порошков необходимого качества. Форма частиц распространенных промышленных металлических порошков далека от сферической, что затрудняет их использование в аддитивных технологиях. Представляет интерес задача сфероидизации частиц порошков, которая может быть реализована путем их высокоскоростного нагрева до температуры плавления. В настоящей работе представлены расчеты, обосновывающие применимость для этой цели мощных источников микроволнового излучения.

Рассмотрим металлическую частицу, облучаемую плоской электромагнитной волной. Для сферического тела мощность $W$, выделяющаяся в нем, определяется на основе решения Ми $[1,2]$

$$
\begin{aligned}
W=- & \pi \frac{\mathbf{E}_{0}^{2}}{k_{0}^{2}} \sqrt{\frac{\varepsilon_{0}}{\mu_{0}}} \sum_{n=1}^{\infty}(2 n+1) \\
& \times\left[\operatorname{Re}\left(a_{n}+b_{n}\right)+\left|a_{n}\right|^{2}+\left|b_{n}\right|^{2}\right]^{2},
\end{aligned}
$$

где $\mathbf{E}_{0}-$ амплитуда электрического поля в волне, $k_{0}=\omega / c-$ волновое число в вакууме, $\omega-$ цикличе-

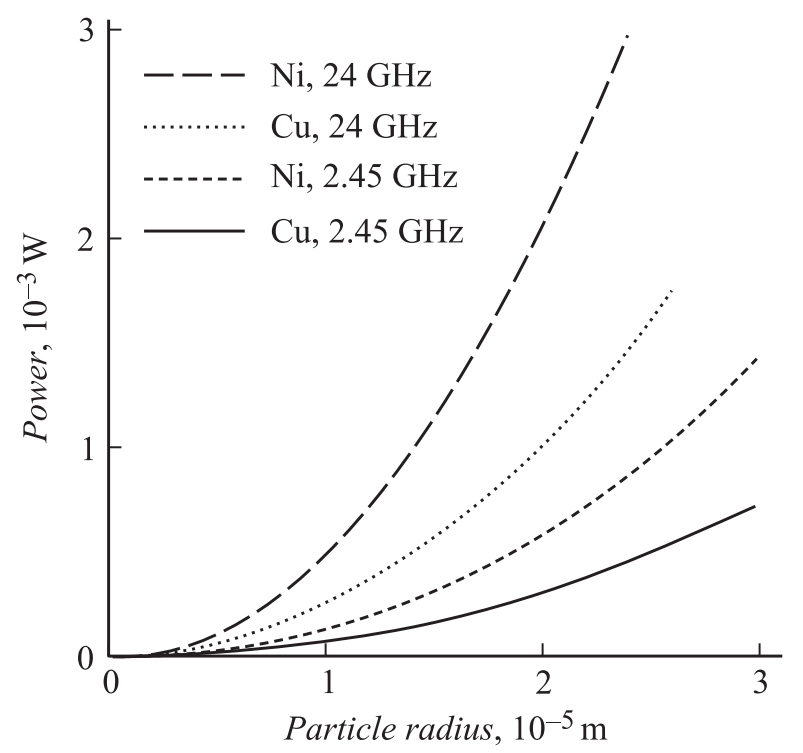

Рис. 1. Мощность, выделяемая в сферической металлической частице, находящейся в поле плоской волны с амплитудой напряженности электрического поля $10 \mathrm{kV} / \mathrm{cm}$, в зависимости от радиуса частицы при комнатной температуре. 
Параметры материалов, использованные при расчетах [3]

\begin{tabular}{l|c|c}
\hline \multicolumn{1}{c|}{ Параметр } & Никель $(\mathrm{Ni})$ & $\mathrm{Mедь}(\mathrm{Cu})$ \\
\hline Плотность, $\mathrm{kg} / \mathrm{m}^{3}$ & 8908 & 38520 \\
\hline Удельная теплоемкость, $\mathrm{J}(\mathrm{kg} \cdot \mathrm{K})$ & 444 & $10^{8} /(1.55+0.0063093(T-273))$ \\
\hline $\begin{array}{l}\text { Электропроводность в зависимости } \\
\text { от температуры } T(\mathrm{~K}),(\Omega \cdot \mathrm{m})^{-1}\end{array}$ & $10^{8} /(6.14+0.027159(T-273))$ & 1356 \\
\hline Температура плавления, $\mathrm{K}$ & 1726 & 0.87
\end{tabular}

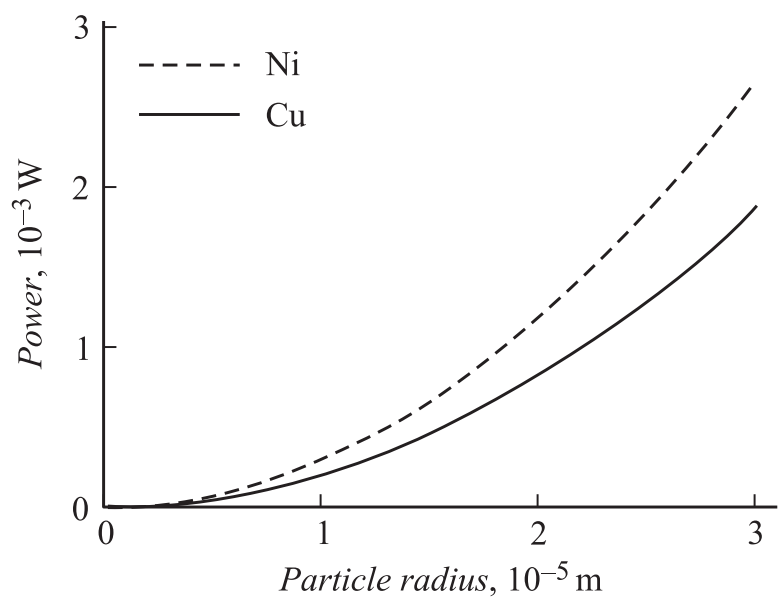

Рис. 2. Мощность, отводимая тепловым излучением от сферической металлической частицы, в зависимости от радиуса частицы при температуре плавления.

ская частота, $c-$ скорость света, $\varepsilon_{0}-$ электрическая постоянная, $\mu_{0}-$ магнитная постоянная,

$$
\begin{gathered}
a_{n}=-\frac{j_{n}(N \rho)\left[\rho j_{n}(\rho)\right]^{\prime}-j_{n}(\rho)\left[N \rho j_{n}(N \rho)\right]^{\prime}}{j_{n}(N \rho)\left[\rho h_{n}^{(1)}(\rho)\right]^{\prime}-h_{n}^{(1)}(\rho)\left[N \rho j_{n}(N \rho)\right]^{\prime}} \\
b_{n}=-\frac{\left.j_{n}(\rho)\left[N \rho j_{n}(N \rho)\right]^{\prime}-N^{2} j_{n}(N \rho)\left[\rho j_{n} \rho\right)\right]^{\prime}}{h_{n}^{(1)}(\rho)\left[N \rho j_{n}(N \rho)\right]^{\prime}-N^{2} j_{n}(N \rho)\left[\rho h_{n}^{(1)}(\rho)\right]^{\prime},}
\end{gathered}
$$

$\rho=k_{0} R, R-$ радиус тела, $N=\sqrt{\varepsilon \mu}, \varepsilon=1+i \sigma / \varepsilon_{0} \omega-$ комплексная диэлектрическая проницаемость материала тела, $\sigma$ - электрическая проводимость материала, $\mu-$ магнитная проницаемость,

$$
\begin{aligned}
& j_{n}(x)=\sqrt{(\pi / 2 x)} J_{n+1 / 2}(x), \\
& h_{n}^{(1)}(x)=\sqrt{(\pi / 2 x)}\left[J_{n+1 / 2}(x)+i Y_{n+1 / 2}(x)\right],
\end{aligned}
$$

$J$ и $Y$ - бесселевы функции первого и второго рода соответственно.

На рис. 1 приведены зависимости мощности, выделяемой в сферических частицах из никеля и меди, от их радиуса для двух стандартных частот излучения, используемых при микроволновой обработке материалов. Параметры материалов, использованные при расчетах в настощей работе, приведены в таблице. На основе данных, приведенных на рис. 1, можно грубо оценить время, необходимое для нагрева частиц до температуры плавления. Без учета потерь тепла можно получить, что,
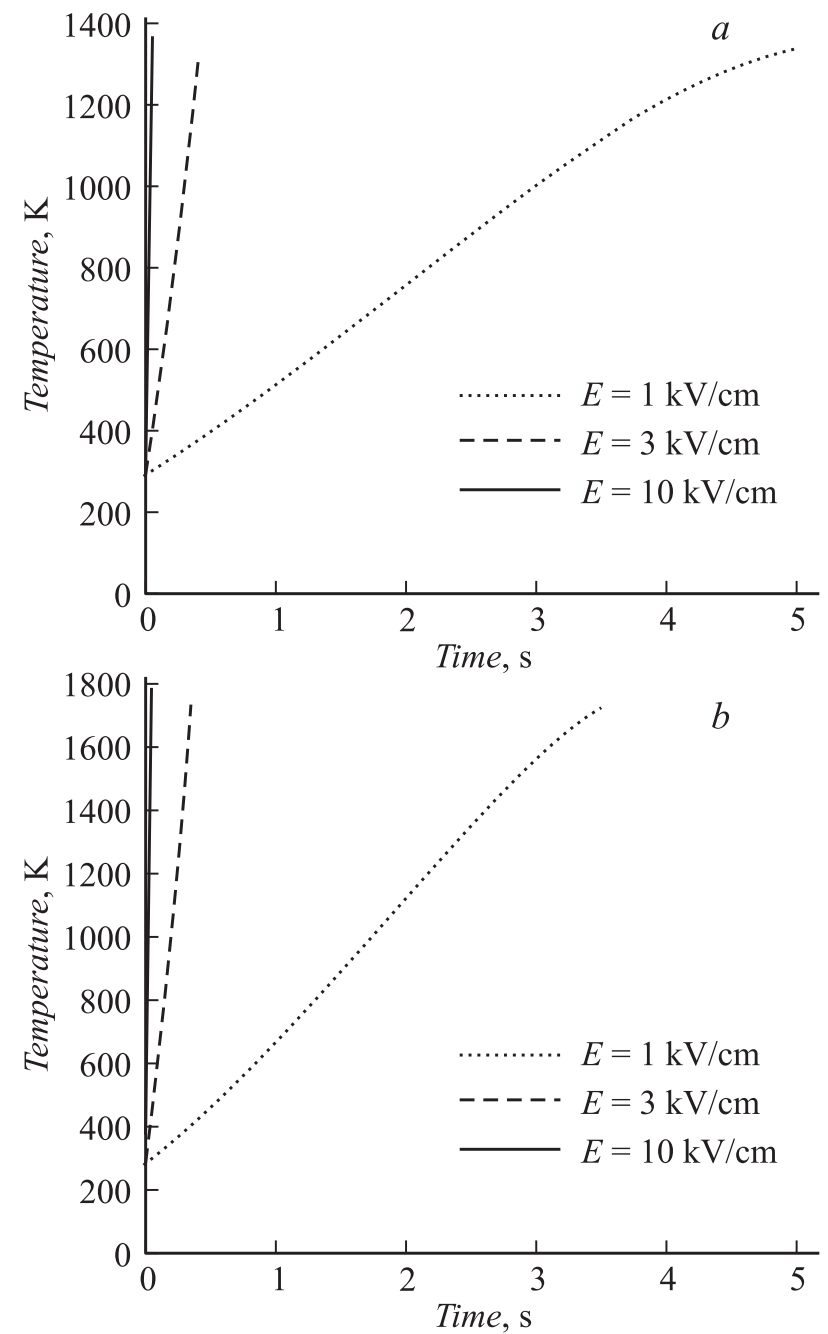

Рис. 3. Изменение температуры порошкового кластера со временем при нагреве в поле плоской электромагнитной волны с различной напряженностью электрического поля: $a-$ медный порошок, $b-$ никелевый порошок. Частота $24 \mathrm{GHz}$, радиус частиц порошка $10 \mu \mathrm{m}$, радиус кластера $5 \mathrm{~cm}$, относительная объемная концентрация частиц 0.01 . 

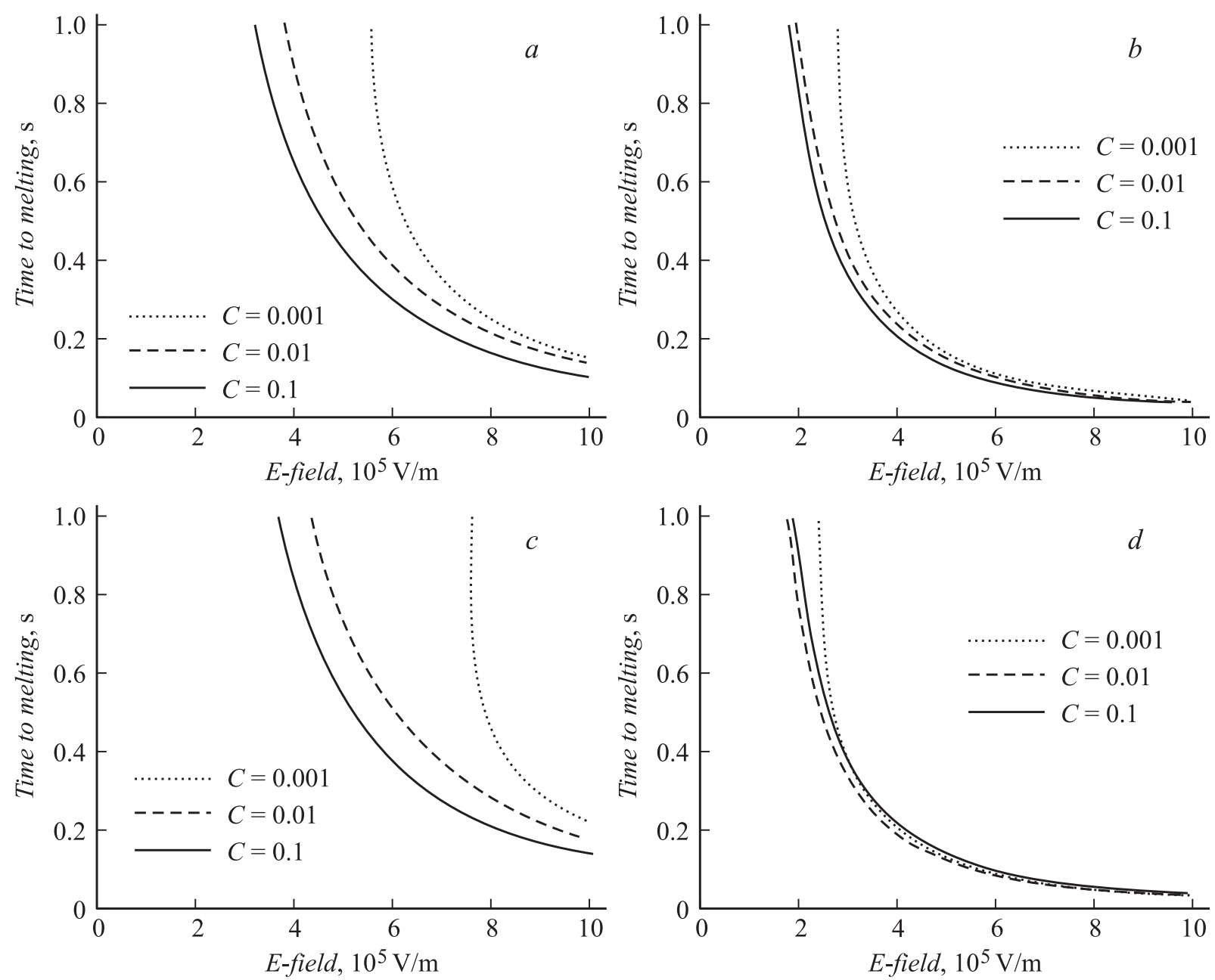

Рис. 4. Время, необходимое для достижения температуры плавления металлических частиц, в зависимости от напряженности электрического поля в падающей волне при различных значениях относительной объемной концентрации частиц: $a-$ медный порошок, частота $2.45 \mathrm{GHz} ; b-$ медный порошок, частота $24 \mathrm{GHz} ; c-$ никелевый порошок, частота $2.45 \mathrm{GHz} ; d-$ никелевый порошок, частота $24 \mathrm{GHz}$. Радиус частиц порошка $10 \mu \mathrm{m}$, радиус кластера $5 \mathrm{~cm}$.

например, при напряженности электрического поля в падающей волне $10 \mathrm{kV} / \mathrm{cm}$ для частиц радиусом $10 \mu \mathrm{m}$ это время варьируется от $0.05 \mathrm{~s}$ (никель на частоте $24 \mathrm{GHz}$ ) до $0.2 \mathrm{~s}$ (медь при $2.45 \mathrm{GHz}$ ). Столь малые значения времени достижения температуры плавления позволяют рассчитывать на возможность реализации сфероидизации металлических порошков при их свободном падении в области интенсивного микроволнового поля. Рассмотрим эту возможность более детально.

При достаточно высоких интенсивностях микроволнового излучения нагрев целесообразно производить в вакууме. В этом случае отвод тепла от частиц происходит благодаря тепловому излучению, интенсивность которого резко возрастает с увеличением температуры. На рис. 2 приведены зависимости мощности, отводимой от медных и никелевых частиц при температуре плавления, от их радиуса. Сопоставление данных, приведенных на рис. 1 и 2, показывает, что отводимая мощность имеет тот же порядок величины, что и мощность, выделяемая в частицах при напряженности электрического поля в падающей волне $10 \mathrm{kV} / \mathrm{cm}$. Это позволяет рассчитывать, что микроволновое излучение с интенсивностью порядка указанной действительно может обеспечить нагрев частиц до температуры плавления.

При практической реализации процесса высокотемпературной сфероидизации металлических частиц микроволновому нагреву должны подвергаться не единичные частицы, а их совокупности (кластеры), имеющие макроскопические размеры. При этом благодаря значительной оптической толщине таких кластеров теплоотвод за счет теплового излучения в расчете на одну частицу будет меньше, чем в случае единичных частиц. Действительно, при малом числе частиц полная мощность излучения будет определяться произведением мощности излучения от одной частицы на число частиц, а при большом числе частиц в кластере мощность излучения будет определяться площадью поверхности кластера как целого, а не суммой площадей поверхностей отдельных частиц. Из двух величин (суммарная мощность излучения всех частиц и мощность излучения с поверхности кластера) 
реализовываться будет всегда меньшая. Если при заданной концентрации частиц увеличивать размеры кластера, то сначала его мощность излучения будет расти пропорционально числу частиц, т.е. пропорционально кубу радиуса (кластер будет иметь малую оптическую толщину), а затем рост мощности излучения замедлится (мощность излучения станет пропорциональна площади поверхности кластера - случай большой оптической толщины). Количество частиц радиуса $a$ в кластере радиуса $R$ при относительной объемной концентрации частиц $C$ равно $C(R / a)^{3}$. Сумма мощностей теплового излучения от отдельных частиц составляет

$$
P_{1}=C(R / a)^{3} 4 \pi a^{2} \varepsilon \sigma_{S B}\left(T^{4}-T_{0}^{4}\right),
$$

где $\varepsilon-$ степень черноты, $\sigma_{S B}-$ постоянная Стефана-Больцмана, $T$ - температура, $T_{0}$ - температура окружающей среды. Мощность, излучаемая с поверхности кластера как целого, равна

$$
P_{2}=4 \pi R^{2} \varepsilon \sigma_{S B}\left(T^{4}-T_{0}^{4}\right)
$$

При выполнении условия $P_{1}>P_{2}$, т.е. при $C R / a>1$, будут реализовываться потери тепла излучением с поверхности кластера как целого, что означает уменьшение теплоотвода в расчете на одну частицу. При микронных размерах частиц и сантиметровых радиусах кластера это условие будет обычно выполнено, во всяком случае для практически разумных (не слишком малых) значений концентрации частиц $C$.

Расчет мощности, выделяемой в кластерах, концентрация частиц в которых не мала, должен выполняться с учетом взаимного влияния частиц. С этой целью можно представить кластер состоящим из однородного вещества, характеризуемого эффективными свойствами, которые определяются в рамках тех или иных моделей. Поглощение энергии микроволнового излучения в металлических частицах происходит за счет одновременного действия двух механизмов: непосредственного возбуждения электрических токов в частицах электрической компонентой поля электромагнитной волны и возбуждения вихревых электрических токов магнитной компонентой поля. Потери первого (электрического) типа пропорциональны мнимой части эффективной диэлектрической проницаемости вещества кластера, потери второго (магнитного) типа - мнимой части эффективной магнитной проницаемости.

Для кластеров металлических частиц с высокой электропроводностью (радиус частиц не мал по сравнению с толщиной скин-слоя $\left.\delta=c \sqrt{2 \varepsilon_{0}(\sigma \omega)}\right)$ эффективную диэлектрическую проницаемость можно получить в рамках приближения эффективной среды, учитывающего волновой характер поля внутри частиц [4]. Уравнение для эффективной диэлектрической проницаемости $\varepsilon_{\mathrm{eff}}$ получается путем усреднения электрического поля $\mathbf{E}$ и электрической индукции $\mathbf{D}$ по объему вещества кластера и имеет вид

$$
2 v_{1} \varepsilon_{\mathrm{eff}}^{2}+\left[(3 C-2) v_{1}+2(3 C-1) \varepsilon_{c} v_{2}\right] \varepsilon_{\mathrm{eff}}+2 v_{2} \varepsilon_{c}=0
$$

где $\varepsilon_{c} \cong i \sigma \varepsilon_{0} \omega$ - диэлектрическая проницаемость электропроводящего материала частиц, $v_{1}=k^{2} a^{2} \sin k a+$ $+k a \cos k a-\sin k a, v_{2}=k a \cos k a-\sin k a, k=k_{0} \sqrt{\varepsilon_{c}} \cong$ $\cong(1+i) / \delta-$ волновое число в материале частиц. На основе аналогичных соображений можно получить уравнение для эффективной магнитной проницаемости $\mu_{\mathrm{eff}}$ вещества кластера. Для немагнитных материалов оно имеет вид

$$
2 v_{1} \mu_{\mathrm{eff}}^{2}+\left[(3 C-2) v_{1}+2(3 C-1) v_{2}\right] \mu_{\mathrm{eff}}+2 v_{2}=0 .
$$

Из двух корней каждого из квадратных уравнений (4) и (5) следует выбирать тот, мнимая часть которого положительна.

Рассмотрим сферический кластер частиц металлического порошка, облучаемый плоской электромагнитной волной. Мощность, выделяемая в таком кластере, рассчитывается по формуле $(1)$, в которой вместо $\varepsilon$ и $\mu$

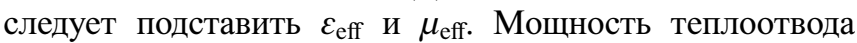
определяется выражением (3). На рис. 3 приведены примеры изменения температуры кластеров со временем для различных значений напряженности поля в падающей волне. Графики оборваны при достижении температуры плавления соответствующего металла.

Как следует из рис. 3, время, необходимое для достижения температуры плавления, быстро убывает с увеличением напряженности поля в падающей волне. На рис. 4,5 приведены результаты расчетов времени, необходимого для достижения температуры плавления, в зависимости от напряженности поля при различных значениях параметров. Из данных, приведенных на рис. 4, следует, что времена достижения температуры плавления, меньшие $1 \mathrm{~s}$ (что можно считать максимально приемлемым временем при реализации нагрева свободно падающих порошков), как правило, реализуются при частоте $2.45 \mathrm{GHz}$ при напряженностях поля в падающей волне $5 \mathrm{kV} / \mathrm{cm}$ и более, а при частоте $24 \mathrm{GHz}$ - при напряженностях поля $2 \mathrm{kV} / \mathrm{cm}$ и более. Из рис. 5 следует, что нагрев кластеров, содержащих более крупные частицы, при прочих равных условиях происходит медленнее и/или требует большей интенсивности излучения.

На рис. 6 приведены зависимости времени, необходимого для достижения температуры плавления, от частоты микроволнового излучения. Из данных, представленных на рис. 6, следует, что эффективность нагрева существенно возрастает при увеличении частоты. Для практически реализуемых размеров порошковых кластеров (радиус порядка нескольких сантиметров) использование излучения частоты $24 \mathrm{GHz}$ является предпочтительным по сравнению с излучением частоты $2.45 \mathrm{GHz}$. Дальнейшее увеличение частоты не приводит к значительному увеличению эффективности нагрева. 

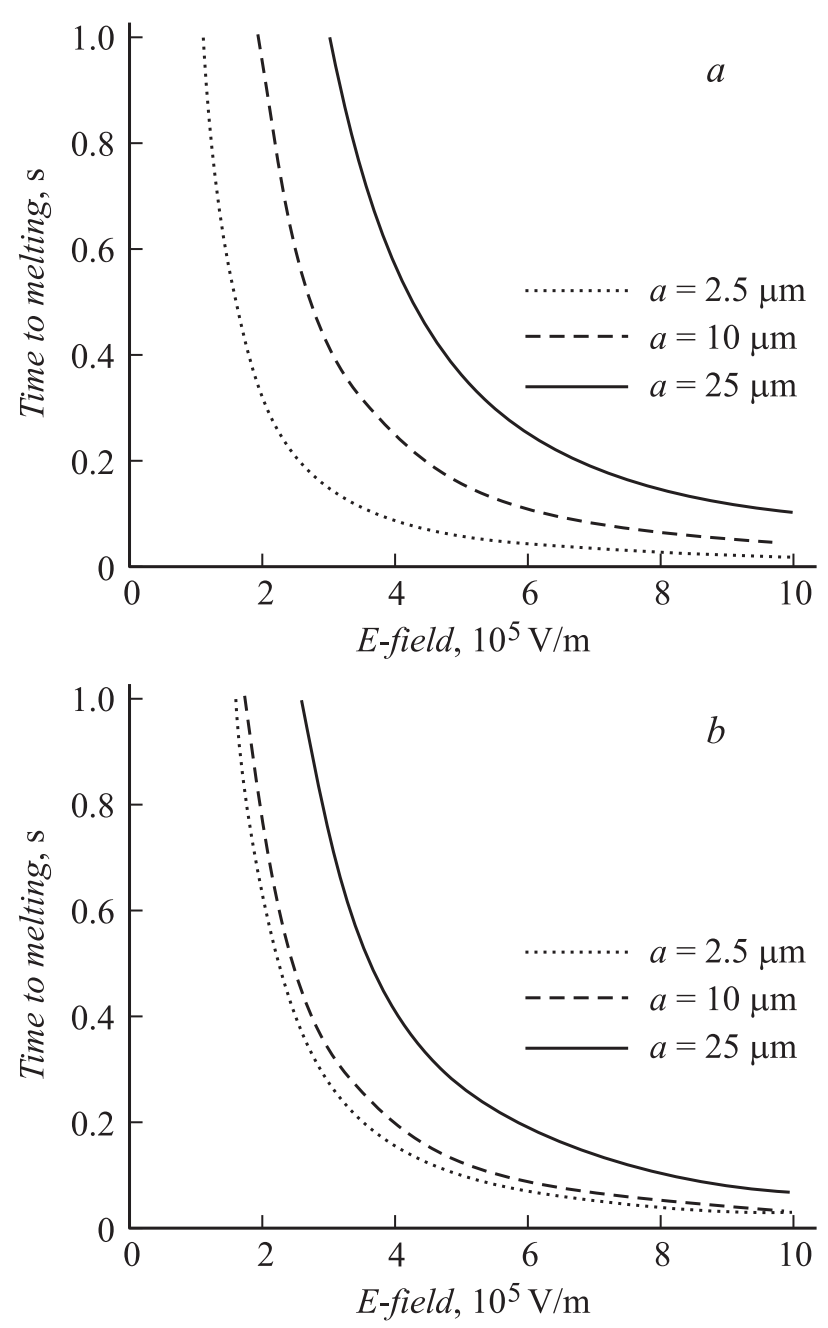

Рис. 5. Время, необходимое для достижения температуры плавления металлических частиц, в зависимости от напряженности электрического поля в падающей волне при различных значениях радиуса частиц порошков: $a-$ медный порошок; $b-$ никелевый порошок. Частота $24 \mathrm{GHz}$, радиус кластера $5 \mathrm{~cm}$, относительная объемная концентрация частиц 0.01 .

На рис. 7 приведены зависимости времени, необходимого для достижения температуры плавления, от радиуса порошкового кластера. Из данных, представленных на рис. 7, следует, что эффективность нагрева снижается, когда размер кластера существенно меньше длины электромагнитной волны. Это проявляется в случае нагрева небольших кластеров (радиусом в единицы сантиметров) излучением частоты $2.45 \mathrm{GHz}$. В случае использования излучения частоты $24 \mathrm{GHz}$ зависимость времени нагрева от размера кластера в указанном диапазоне несущественна.

Энергетическую эффективность использования микроволнового излучения для нагрева порошковых кластеров можно охарактеризовать величиной сечения поглощения, т.е. отношением мощности, выделяемой в сферическом кластере, к интенсивности излучения в падающей плоской волне. Сечение поглощения целе-
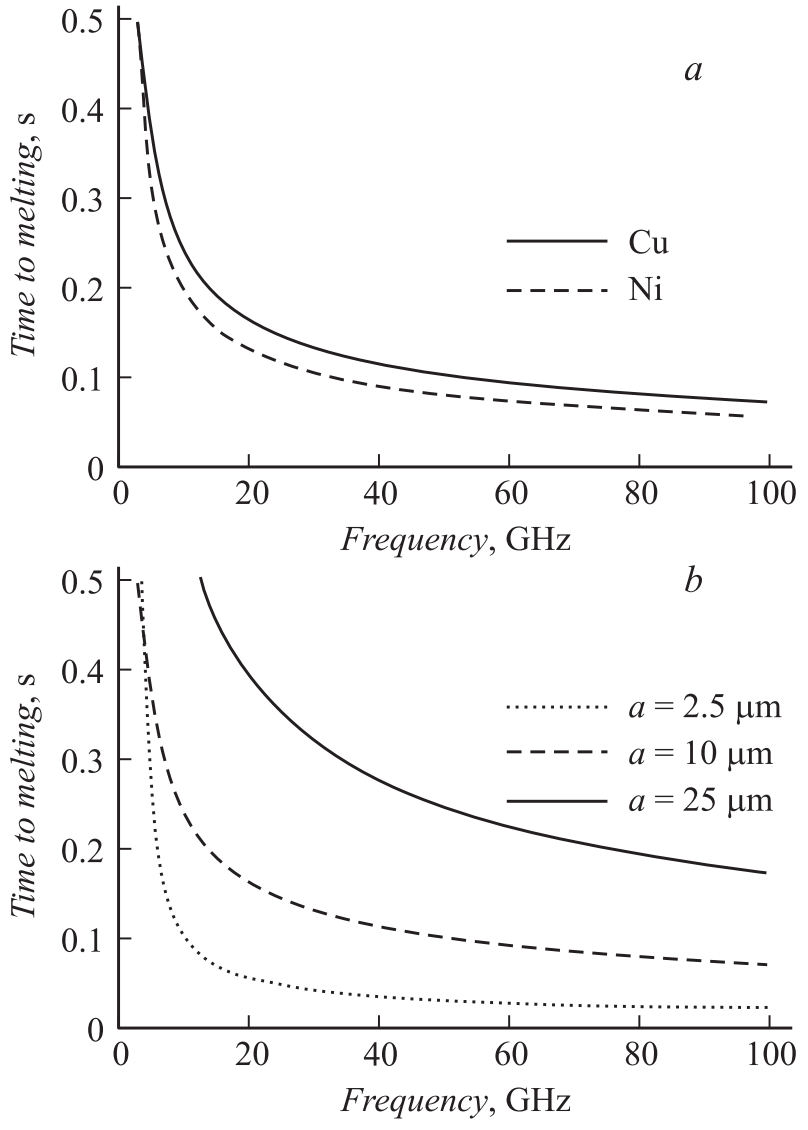

Рис. 6. Время, необходимое для достижения температуры плавления металлических частиц, в зависимости от частоты микроволнового излучения: $a-$ для медных и никелевых частиц радиусом $10 \mu \mathrm{m} ; b$ - для медных частиц трех различных радиусов. Напряженность электрического поля в падающей волне $5 \mathrm{kV} / \mathrm{cm}$, радиус кластера $5 \mathrm{~cm}$, относительная объемная концентрация частиц 0.01 .

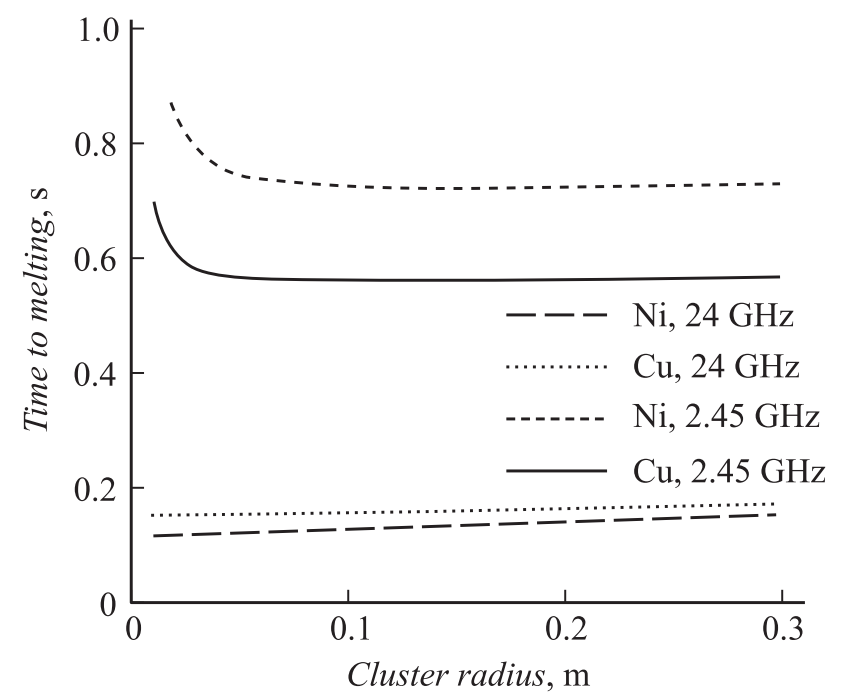

Рис. 7. Время, необходимое для достижения температуры плавления металлических частиц, в зависимости от радиуса порошкового кластера для медных и никелевых частиц при двух различных частотах. Напряженность электрического поля в падающей волне $5 \mathrm{kV} / \mathrm{cm}$, относительная объемная концентрация частиц 0.01 , радиус частиц порошка $10 \mu \mathrm{m}$. 


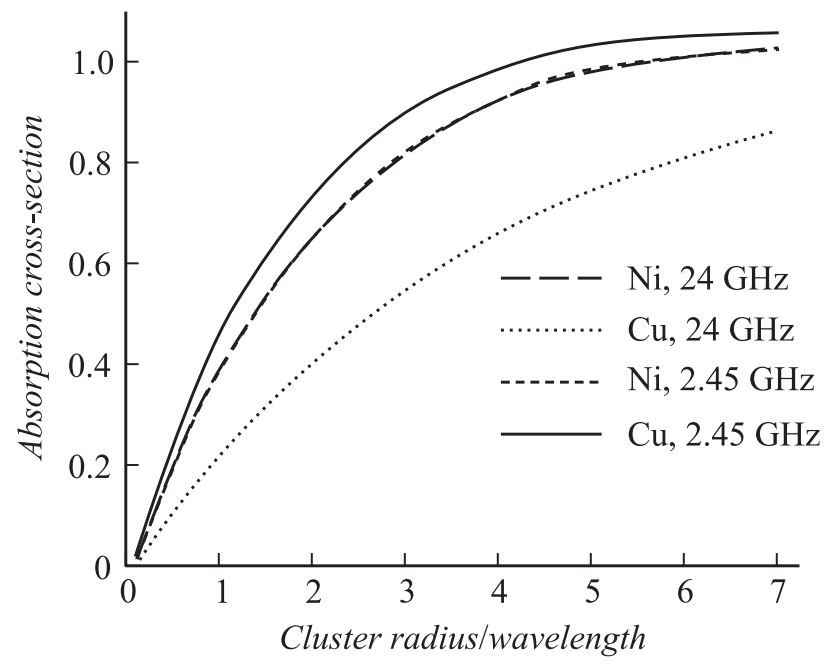

Рис. 8. Сечение поглощения, нормированное на площадь большого круга сферического кластера, в зависимости от радиуса порошкового кластера, отнесенного к длине волны, для двух значений частоты для медных и никелевых частиц при температуре плавления. Относительная объемная концентрация частиц 0.1 , радиус частиц порошка $10 \mu \mathrm{m}$.

сообразно нормировать на площадь большого круга сферического кластера. На рис. 8 приведены зависимости такого нормализованного сечения поглощения от радиуса кластера, отнесенного к вакуумной длине волны излучения. Из рис. 8 следует, что с ростом размера кластера нормализованное сечение поглощения монотонно возрастает и выходит на предельное значение, соответствующее полному поглощению излучения в кластере. Отметим, что выбранное для расчетов (рис. 3-6) реалистическое значение радиуса порошкового кластеpa $5 \mathrm{~cm}$ на частоте $24 \mathrm{GHz}$ соответствует 4 длинам волн и обеспечивает достаточно высокую эффективность нагрева. На частоте $2.45 \mathrm{GHz}$ радиус такого кластера составляет всего около 0.4 длины волны, и энергетическая эффективность его нагрева невелика, поскольку большая часть излучения проходит сквозь кластер без поглощения.

Таким образом, представленные в настоящей работе результаты подтверждают возможность использования нагрева интенсивным микроволновым излучением для высокоскоростного расплавления металлических порошковых частиц. При напряженностях поля в падающей волне порядка $2 \mathrm{kV} / \mathrm{cm}$ на частоте $24 \mathrm{GHz}$ и $5 \mathrm{kV} / \mathrm{cm}$ на частоте $2.45 \mathrm{GHz}$ время нагрева до температуры плавления не превышает $1 \mathrm{~s}$. Энергетическая эффективность микроволнового нагрева достигает высоких значений при размерах порошковых кластеров, превышающих несколько длин волн излучения в вакууме. Высокоскоростной микроволновый нагрев, таким образом, является перспективным методом для реализации сфероидизации металлических частиц, необходимой для производства высококачественных порошков для аддитивных технологий. Использование для этой цели микроволнового излучения миллиметрового диапазона, начиная со стандартной частоты $24 \mathrm{GHz}$, является предпочительным.

Данные исследования частично поддержаны грантом Российского научного фонда № 15-12-00046.

\section{Список литературы}

[1] Mie G. // Annalen der Physik. 1908. Vol. 330. P. 377-445.

[2] Stratton, J.A. Electromagnetic Theory. NY:: McGraw-Hill, 1941. $615 \mathrm{p}$.

[3] Физические величины: Справочник / Под ред. И.С. Григорьева, Е.3. Мейлихова. М.: Энергоатомиздат, 1991. 1232 с.

[4] Rybakov K.I., Semenov V.E. // IEEE Transactions on Microwave Theory and Techniques, 2017. Vol. 65. P. 14791487. 